\title{
Encuesta sobre docencia online en las Facultades de Enfermería y Medicina de la Universidad de Murcia durante COVID-19.
}

\section{Survey on virtual teaching in the Faculties of Nursing and Medicine of the University of Murcia during COVID-19.}

\author{
Rojo Martínez $\mathrm{JM}^{\mathbf{1}}$, Conesa Erragbaoui AG $^{\mathbf{1}}$, Vera Villalba $\mathbf{J}^{\mathbf{1}}$, García García \\ MA $^{1}$, Martínez de Rituerto Rey $\mathbf{P}^{2}$, Cano Dólera $\mathbf{B}^{2}$, Silvente Bernal $\mathbf{S}^{3}$, Bernal \\ García $\mathbf{A}^{3}$ \\ ${ }^{1}$ Consejo de Estudiantes de la Universidad de Murcia, ceum@um.es, josemiguel.rojo@um.es, \\ amalguzman.conesae@um.es, javier.verav@um.es, miguelangel.garcia18@um.es \\ 2 Delegación de Estudiantes de la Facultad de Enfermería de la Universidad de Murcia, \\ daenfe@um.es, p.martinezrituertorey@um.es, beatriz.canod@um.es \\ ${ }^{3}$ Delegación de Estudiantes de la Facultad de Medicina de la Universidad de Murcia, \\ damedi@um.es, sara.silventeb@um.es.antonio.bernalg@um.es
}

Recibido: 17 de abril de 2020; Aceptado: 4 de mayo de 2020; Publicado: 5 de mayo de 2020

\begin{abstract}
Resumen: Presentamos los resultados de una encuesta sobre docencia virtual realizada por las Delegaciones de Estudiantes de Enfermería y Medicina de la Universidad de Murcia. El trabajo ha sido llevado a cabo en el período de suspensión de la docencia presencial en la Universidad de Murcia, previo al período de vacaciones de primavera, a consecuencia de la pandemia de COVID-19. En general, la percepción del estudiantado de las Facultades de Enfermería y Medicina, similar al del resto de sus compañeros en los demás centros de la Universidad, dista de ser satisfactoria, con un aumento de la carga de trabajo percibida y una gran variabilidad en las modalidades usadas para suplir la docencia presencial.
\end{abstract}

Palabras clave: Docencia Virtual; Encuesta, Estudiantes, Enfermería; Medicina

\begin{abstract}
We present the results of a survey on virtual teaching carried out by the Delegations of Nursing and Medicine Students of the University of Murcia (Spain). It has been carried out in the period of suspension of classroom teaching at the University of Murcia, prior to the spring break period. In general, the perception of the students of the Faculties of Nursing and Medicine is far from being satisfactory. There is great variability in the modalities used to replace face-to-face teaching. The students have perceived also more workload from the professors.
\end{abstract}

Keywords: Virtual Teaching; Survey; University Students; Nursing; Medicine

\section{Introducción}

A partir del 13 de marzo, con la declaración del Estado de Alarma en España, todas las actividades presenciales en la Universidad cesaron bruscamente y obligaron a los profesores a pasar de forma inmediata a un sistema de docencia en línea (online) con el que seguir impartiendo docencia a sus alumnos.

Dada la situación excepcional, la Universidad de Murcia, siguiendo las instrucciones de la Consejería de Universidades, tomó el pasado 12 de marzo la decisión de suspender la docencia presencial universitaria. Es por esto que, desde el pasado 16 de marzo, se implementó la docencia online en esta Universidad. Desde el 
Consejo de Estudiantes de la Uiversidad de Murcia (CEUM), considerando que el desarrollo de una modalidad especial como es la online, requiere evaluar permanentemente sus aspectos derivados para detectar ámbitos de mejora, se ha impulsado una encuesta a todos los estudiantes para poder evaluar las inquietudes y quejas de los estudiantes.

En este artículo presentamos los resultados de esta encuesta sobre docencia online, específicamentre los de los Estudiantes de las Facultades de Enfermería y Medicina, dentro de la encuesta global llevada a cabo por el Consejo de Estudiantes de la Universidad (CEUM). Mientras que las respuestas de la Facultad de Enfermería son únicamente del Grado en Enfermería, las de la Facultad de Medicina corresponden a estudiantes de los 4 Grados que esta Facultad gestiona, Farmacia, Fisioterapia, Medicina y Odontología.

En la encuesta global han participado 8303 estudiantes de toda la Universidad, lo que supone un $25,7 \%$ del censo estudiantil de la UMU. Una cifra muy importante si la comparamos en perspectiva, por ejemplo, con el porcentaje de participación en las últimas elecciones rectorales -el último gran proceso global de participación que implicaba a los estudiantes- en el que participó un 15,27\% del censo total de los estudiantes.

\section{Encuesta sobre docencia online en el Grado en Enfermería.}

A continuación, como cortesía del CEUM y de la Delegación de Estudiantes de Enfermería, presentamos los resultados de la encuesta que se realizó entre sus alumnos (26 y 29 de marzo de 2020) y que formaba parte de la encuesta que se llevó a cabo en toda la UMU. Las principales conclusiones globales de este estudio fueron presentadas a través de un comunicado del CEUM, el 30 marzo de 2020 (1). La Facultad de Enfermería dispone de un total de 864 matrículas, de acuerdo con las cifras de oferta oficiales de la Universidad (2). No se dispone de información del número de estudiantes de Posgrado.

Respondieron 279 estudiantes de Grado y 5 de Posgrado. La figura 1 muestra el número y porcentaje de las respuestas obtenidas según el nivel Grado en Enfermería cursado. En la figura 2 se observa que la opinión de los estudiantes de $1^{\text {o }}$ y $2^{\text {o }}$ es mayoritaria en la percepción de que la carga de trabajo ha aumentado.

\section{Procedencia de los estudiantes en Enfermería}

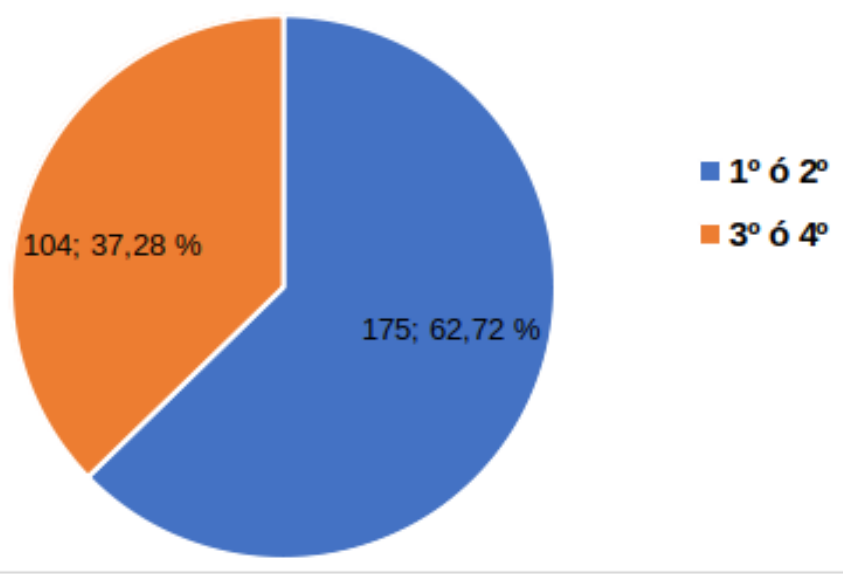

Figura 1. Distribución de las respuestas del estudiantado de Enfermería. 


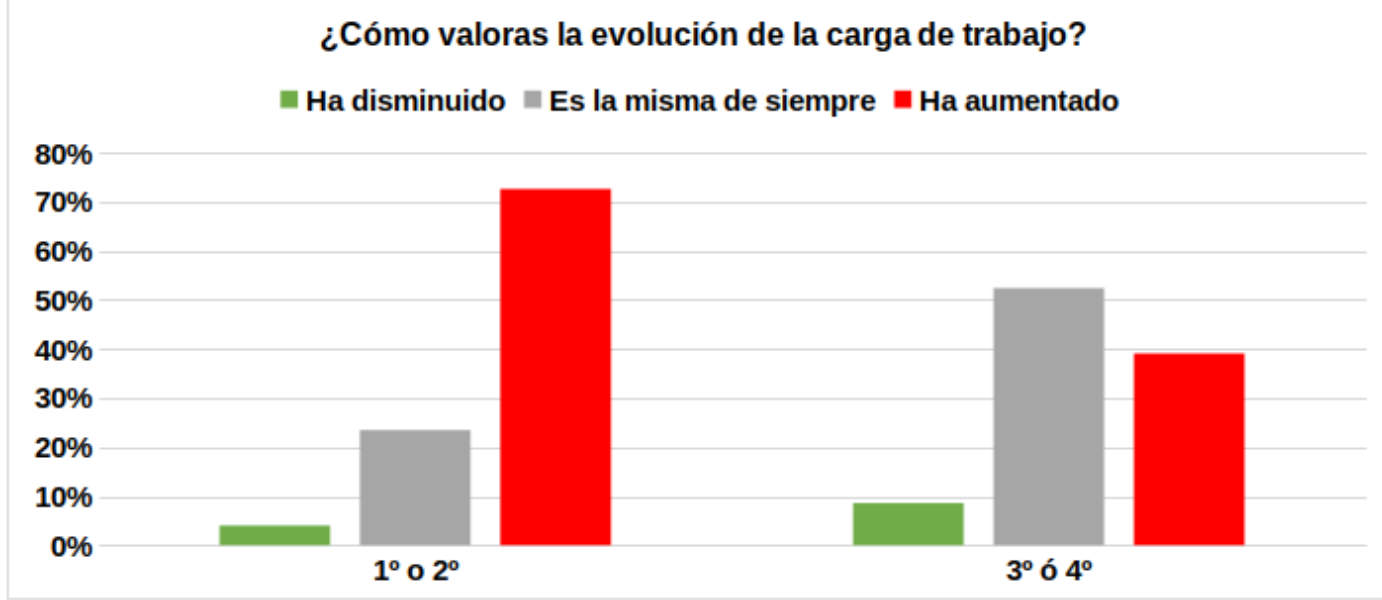

Figura 2. Valoración de la carga de trabajo durante las 3 semanas previas a las vacaciones de primavera en el Grado de Enfermería $(\mathrm{N}=279)$

En la figura 3 se representan las distintas respuestas que el profesorado ha tenido en respuesta al cambio de docencia presencial a virtual. Se observa que, en un gran porcentaje, el profesorado de $3^{\circ}$ y y $4^{\circ}$ realiza videoconferencias y las graba, con un porcentaje mínimo en el que no ha habido interacción con el docente. La figura 4 muestra que, según los estudiantes, la actuaciones inadecuada del profesorado más frecuente fue la de impartir docencia a una hora distinta de la habitual. Finalmente, la valoración global de la docencia online durante este período se muestra en la figura 5. En $1^{\circ}$ y $2^{\circ}$ curso, un $75 \%$ la valora regular o negativamente, mientras que un poco más de un $20 \%$ lo hace positivamente. Este porcentaje aumenta mucho en los estudiantes de los cursos clínicos, hasta casi un $75 \%$.
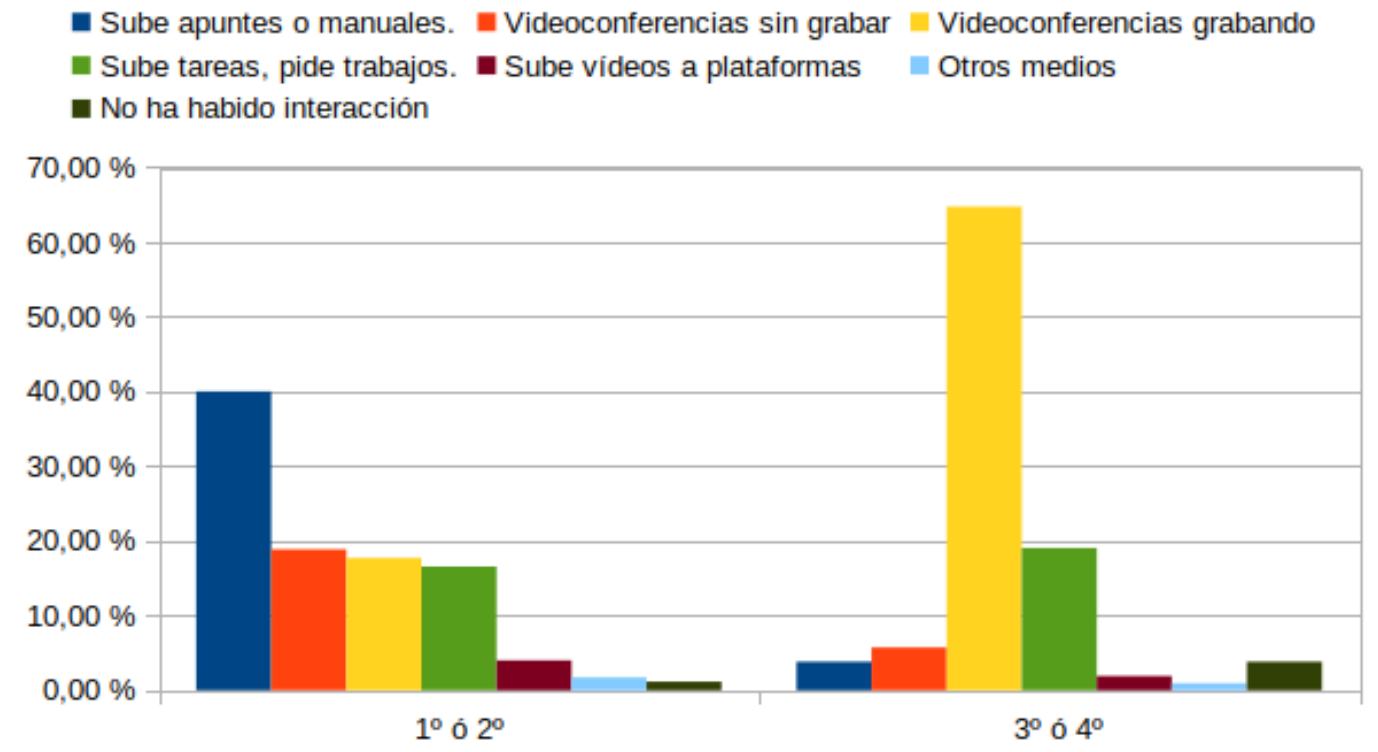

Figura 3. Actividades del profesorado de la Facultad de Enfermería, según la respuesta de sus alumnos. 


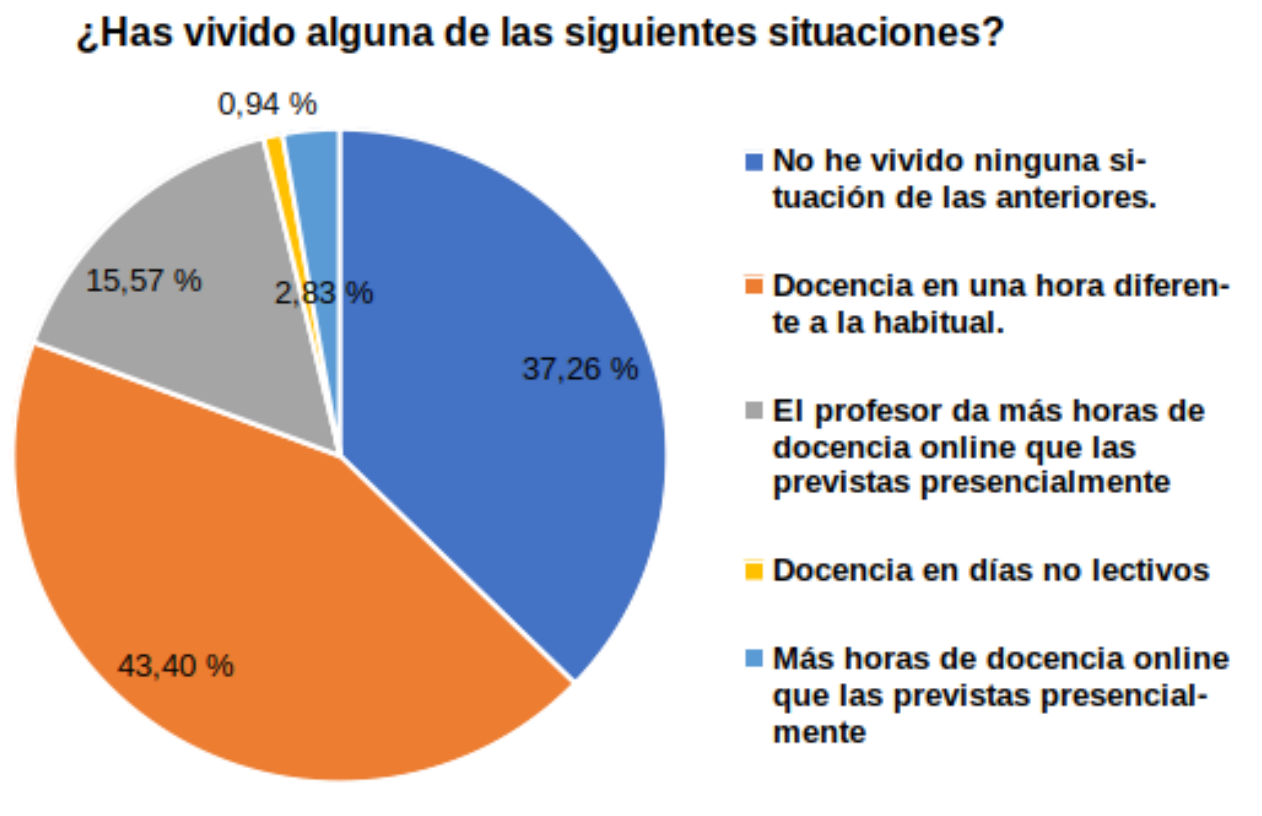

Figura 4. Algunas situaciones anormales detectadas en el Grado de Enfermería.

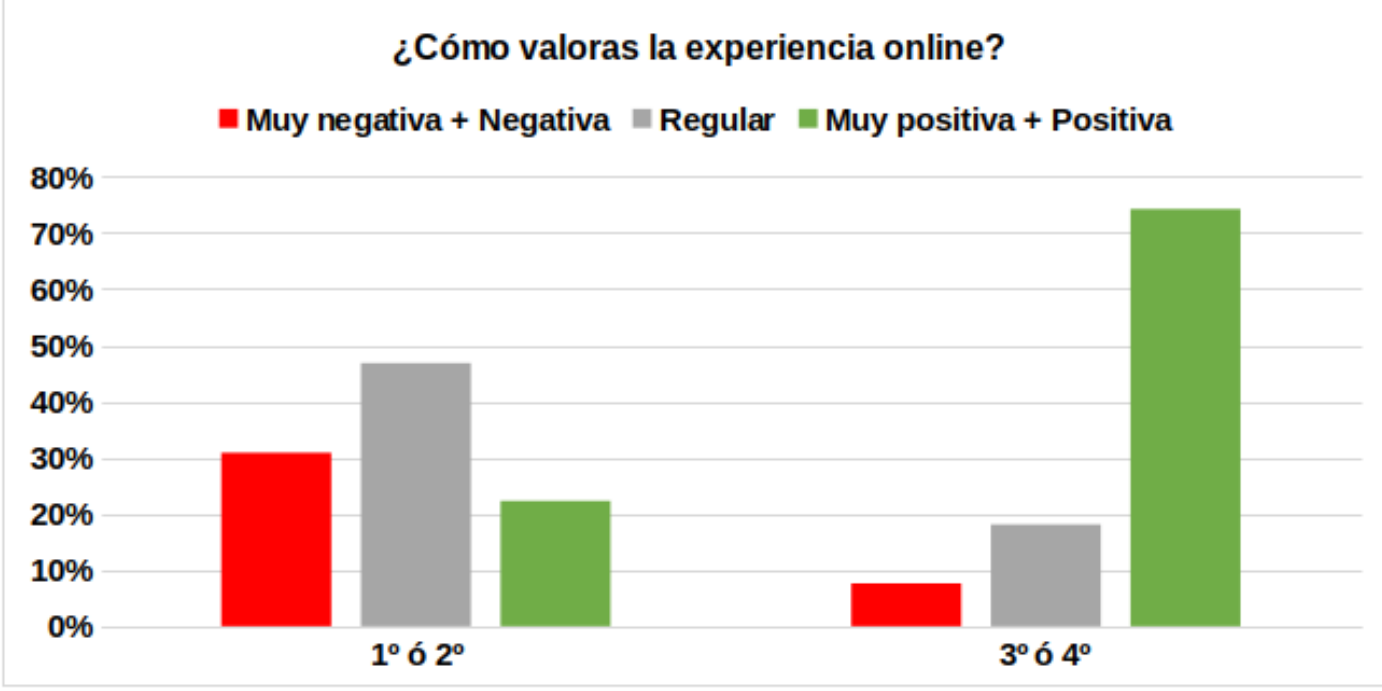

Figura 5. Valoración global de la docencia online en el Grado de Enfermería.

\section{Encuesta sobre docencia online en la Facultad de Medicina.}

Igual que en el punto anterior, como cortesía del CEUM y de la Delegación de Estudiantes de Medicina, se presentan los resultados de la encuesta que se desarrolló en la Facultad de Medicina durante el 26 y 29 de marzo de 2020 y que formaba parte de la encuesta en toda la UMU. Como se ha comentado antes, la Facultad gestiona 4 Grados, Farmacia, Fisioterapia, Medicina y Odontología y los datos no se han podido desagregar en ellos ya que no se preguntó a los encuestados sobre el Grado que estudiaban. En total, la Facultad tiene un total de 1950 matrículas, de acuerdo con las cifras de oferta oficiales de la Universidad (2). No se dispone de información del número de estudiantes de Posgrado.

El número de estudiantes que respondieron a la encuesta fue de 659, 4 de ellos de Posgrado. En la figura 6, se observa la distribución del estudiantado en los 3 niveles, iniciales, medio y final. La figura 7 muestra que la mayoría del profesorado se ha limitado a poner documentos en el aula virtual o a pedir trabajos, con pocas actividades de vídeos o grabaciones de clases. En la figura 8, se observa que la mayoría del profesorado tuvo actuaciones adecuadas, con un porcentaje alrededor de 
un $30 \%$ de los casos en los que se detectaron algunos problemas, fundamentalmente el impartir la docencia en un horario distinto al habitual. De entre los casos en los que se usó aplicaciones de videoconferencia, la figura 9 muestra el porcentaje de uso de estas aplicaciones en los 3 niveles del Grado. El estudiantado también tiene la percepción de que la carga de trabajo ha aumentado, fundamentalmente en los 4 primeros cursos (figura 10). Finalmente, la percepción global es mayoritariamente regular y negativa con valores de un $78 \%$ en $1^{\circ}-2^{\circ}$, el $88 \%$ en $3^{\circ}-4^{\circ}$ y el $76 \%$ en $5^{\circ}-6^{\circ}$ (figura 11).

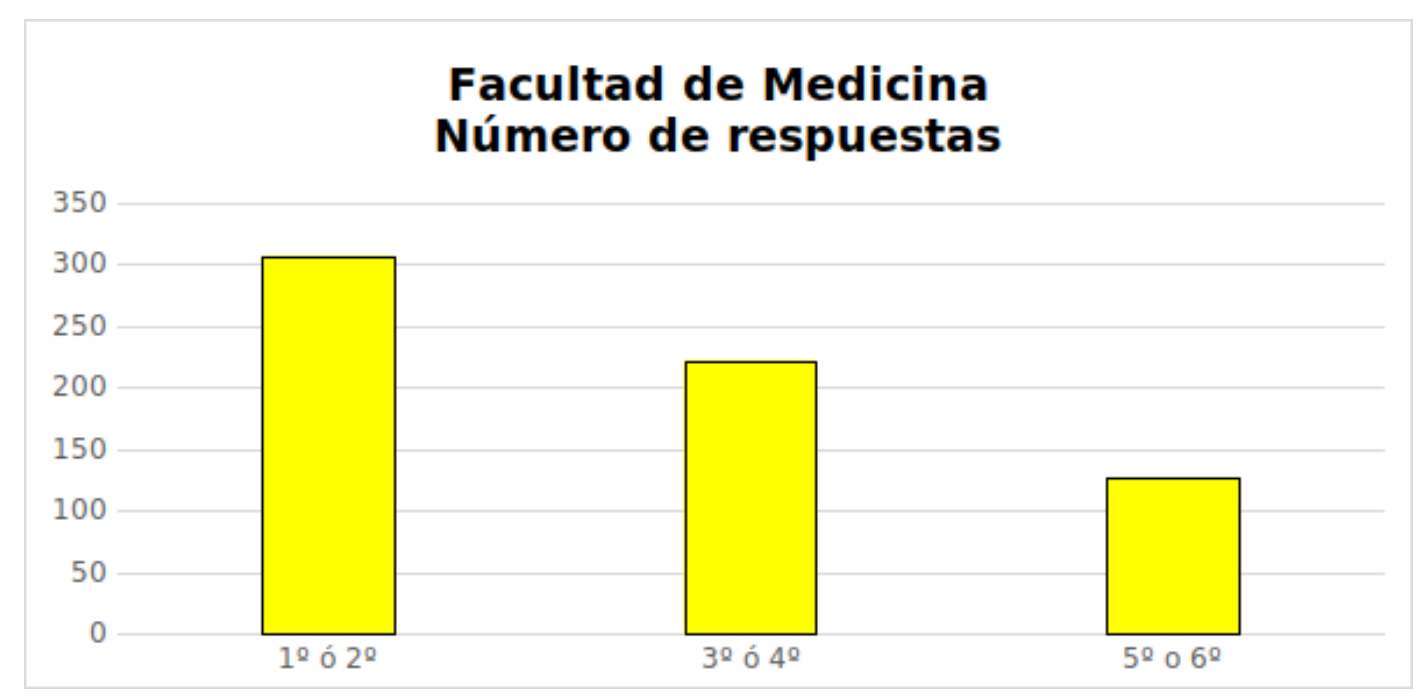

Figura 6. Distribución de las respuestas en los cursos de la Facultad de Medicina

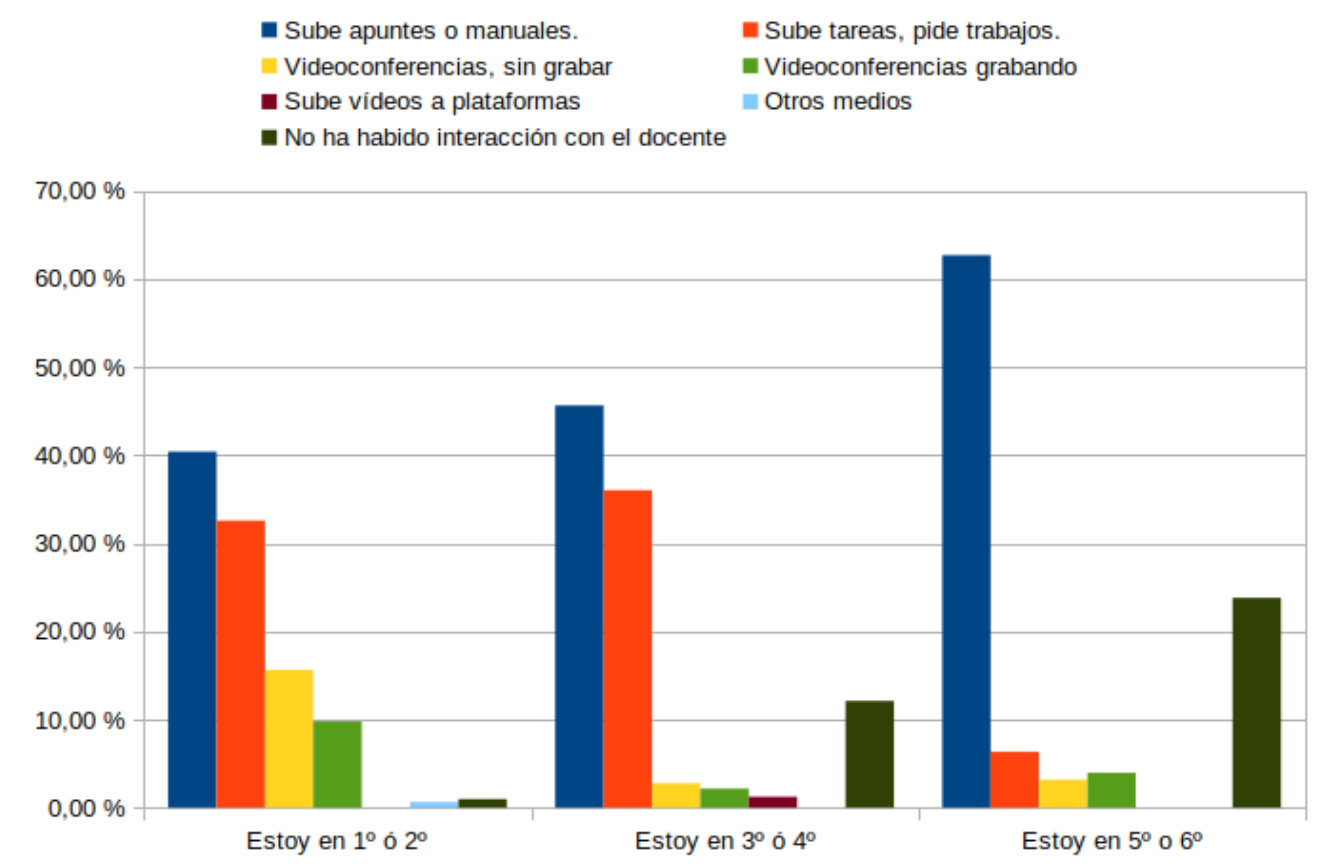

Figura 7. Actividades del profesorado de la Facultad de Medicina según sus estudiantes. 


\section{¿Has vivido alguna de estas situaciones?}

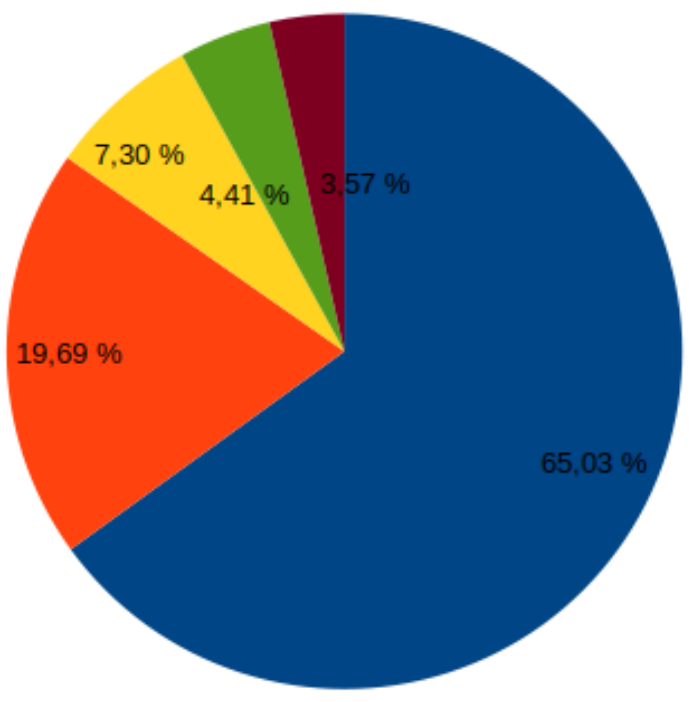

No he vivido ninguna situación de las anteriores.

Docencia en una hora diferente a la habitual.

El profesor da más horas de docencia online que las previstas presencialmente

Docencia en días no lectivos

- Más horas de docencia online que las previstas presencialmente

Figura 8. Algunas situaciones anormales detectadas en la Facultad de Medicina.

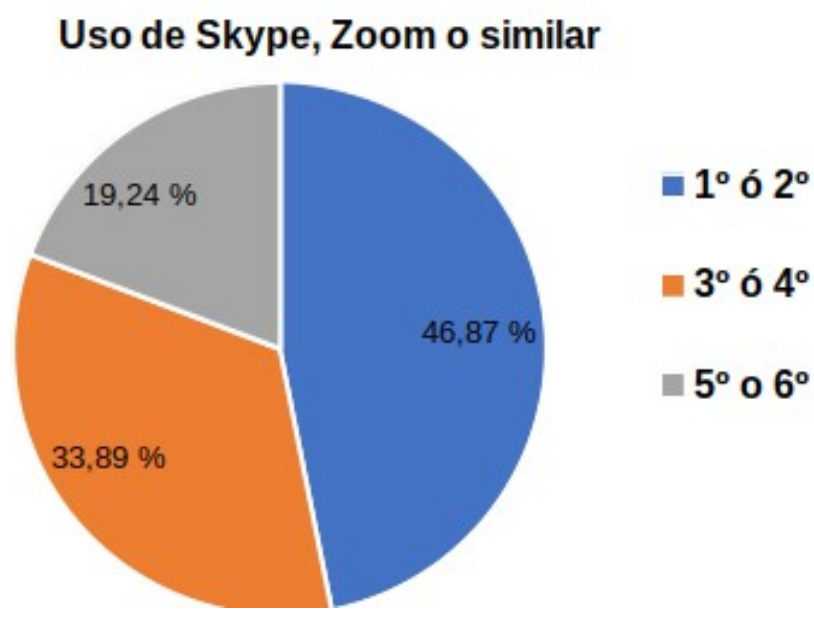

Figura 9. Uso de aplicaciones de videoconferencia

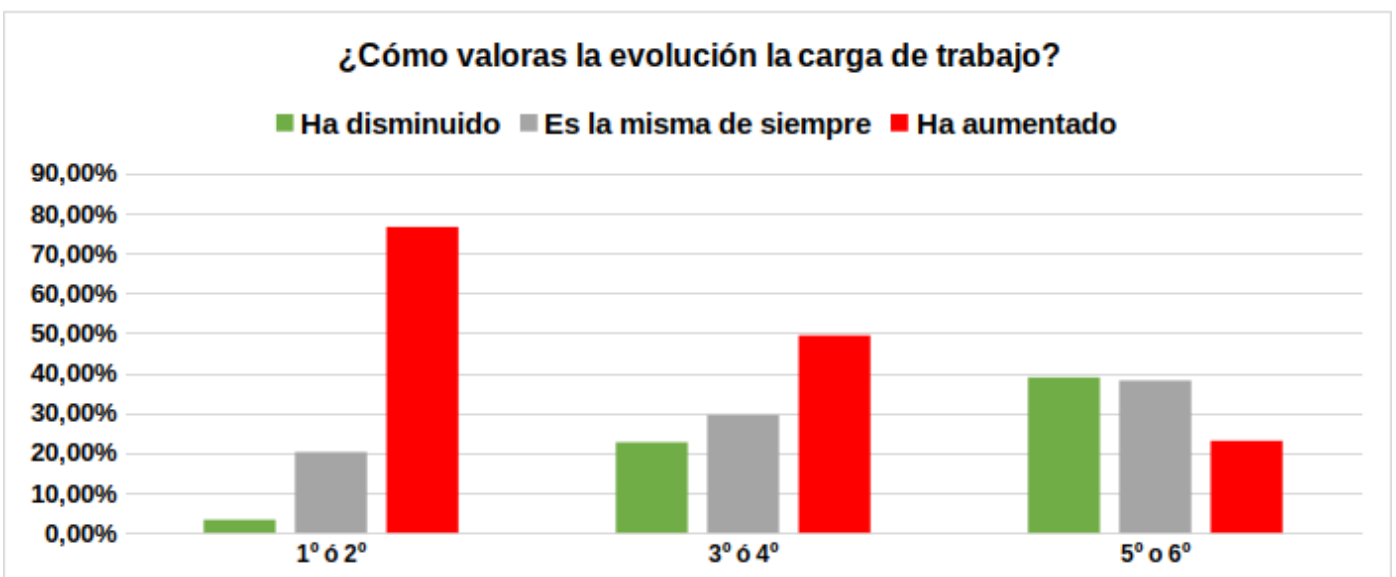

Figura 10. Percepción de la carga de trabajo en la Facultad de Medicina 
Considerando la excepcionalidad de la situación, en líneas generales, ¿cómo valoras la experiencia online?

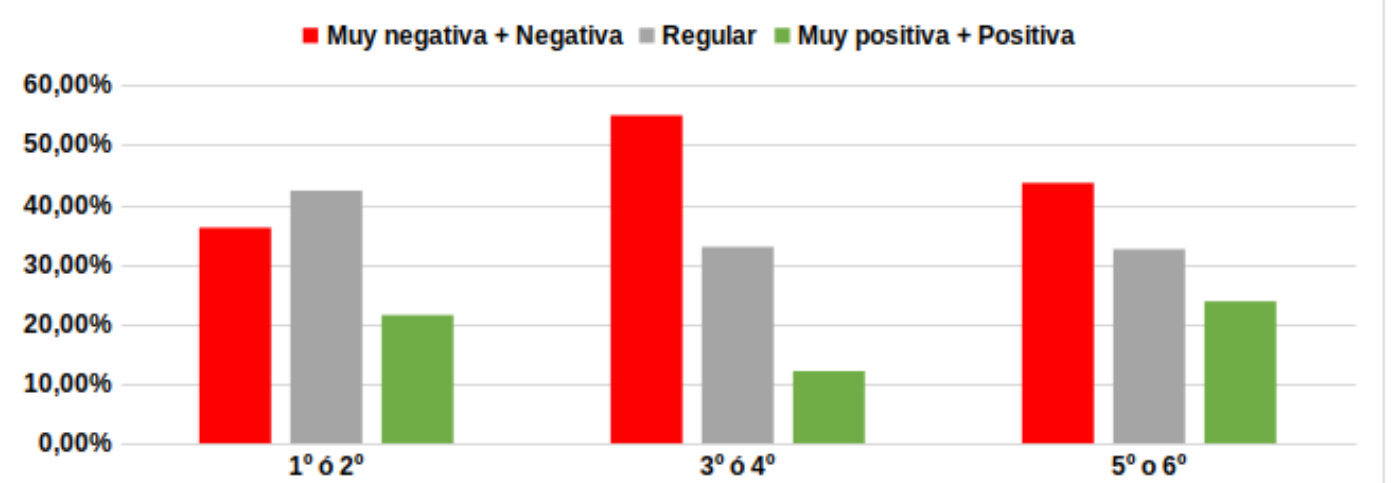

Figura 11. Valoración global de la experiencia online en la Facultad de Medicina.

\section{Discusión.}

Los datos de las Facultades de Enfermería y Medicina son fieles a los resultados globales obtenidos a nivel global en la Universidad de Murcia. Así, según el comunicado del Consejo de Estudiantes (1), se ha detectado un "aumento preocupante de la carga de trabajo, ya que el $67,3 \%$ de los estudiantes afirma que su carga de trabajo ha aumentado estos días". Según el Consejo, esta situación dificulta las condiciones de estudio y preparación de los exámenes finales, que se siguen manteniendo con normalidad. En el citado comunicado, el Presidente del Consejo de Estudiantes de la UM afirma que "el impulsivo establecimiento de trabajos, tareas o clases no supone que la docencia sea mejor, es más, nos parece completamente contraproducente en el contexto actual.

En la encuesta global, la mayoría de los encuestados $(44,6 \%)$ afirma que el docente "sube tareas o pide entrega de trabajos" mientras que un $22,1 \%$ que afirma que "simplemente sube nuevos recursos como apuntes o manuales". Los estudiantes entienden que "la Universidad de Murcia es una Universidad presencial, por lo que no siempre se cuenta con los medios precisos para realizar una docencia online en plenitud. No obstante, nos parece preocupante reducir esta modalidad a subir manuales o mandar tareas sin impartir las explicaciones o lecciones". Y concluye, "los estudiantes requieren explicaciones complementarias al mero temario. Si estas no se dan, por las circunstancias que fueran, esos contenidos deben reconsiderarse a la hora de la evaluación de la asignatura". Además, recuerdan que "el exceso de carga de trabajo derivado ligado a la falta de explicaciones complementarias a los documentos proporcionados, está imposibilitando el estudio, así como la necesaria concentración, descanso y salud mental de los estudiantes en una situación ya de por sí complicada".

Por otra parte, el Consejo de Estudiantes también ha felicitado a los docentes que están desarrollando videoconferencias, subiendo audios o vídeos a la plataforma, porque "realmente responde con acierto a las necesidades del momento" y al equipo del Área de Tecnologías de la Información y las Comunicaciones Aplicadas (ATICA) de la Universidad por su implicación en el correcto desarrollo de la docencia online.

\section{Conclusiones}

- En general, la percepción del estudiantado de las Facultades de Enfermería y Medicina no es satisfactoria habiéndose detectado una baja actividad de actividades de videoconferencia.

- Los estudiantes tienen la percepción de que la carga de trabajo ha aumentado, fundamentalmente en los cursos iniciales y esto parece estar relacionado con el envío de más documentos o apuntes y con la petición de trabajos. 
- Los estudiantes también han felicitado a los docentes que están desarrollando videoconferencias, subiendo audios o vídeos a la plataforma, porque "realmente responde con acierto a las necesidades del momento".

- También se felicita al equipo del Área de Tecnologías de la Información y las Comunicaciones Aplicadas (ATICA) de la Universidad de Murcia por su implicación en el correcto desarrollo de la docencia online.

Declaración de conflicto de interés: Los autores declaran no tener ningún conflicto de interés.

\section{Referencias}

1. Comunicado del CEUM: https://www.murcia.com/region/noticias/2020/03/30-losestudiantes-evaluan-la-docencia-online-de-la-universidad-de-murcia-y-piden-medidassocioeconomicas.asp (consultado el 24 de abril de 2020).

2. Oferta de plazas de Grado, Universidad de Murcia (consultado el 24 de abril de 2020).

2020 por los autores. Enviado para su posible publicación en acceso abierto bajo los términos y condiciones de la licencia Creative Commons Attribution (CC BY) (http://creativecommons.org/licenses/by/4.0/). 